\title{
TRANS-PACIFIC-PARTNERSHIP: EL COMPLEJO CAMINO DEL ACUERDO DEL SIGLO XXI
}

\author{
Rodrigo Cuevas*
}

\begin{abstract}
Resumen
El presente artículo estudia las características del principal proceso negociador de Acuerdos Comerciales Regionales que se está llevando actualmente a cabo en el mundo y sus posibles efectos en futuras negociaciones de este tipo. A partir de las características del caso analizado, un contexto de proliferación de acuerdos de este tipo y de estancamiento de las negociaciones en el ámbito multilateral, se argumenta que más que un acuerdo entre 12 países, este proceso sería un acuerdo de EE.UU. para el Asia-Pacífico.

Clasificación JEL: F13 - F15

Palabras clave: acuerdos comerciales regionales, procesos negociadores, bloques comerciales, libre comercio.
\end{abstract}

\begin{abstract}
This paper research the most important negotiation process of Regional Trade Agreements (RTA) that has been developing actually, which one could take effect in how RTA would be negotiated in the future. From the analyzing of the specifics characteristics of this case, in the context of a explosive increment of RTA and the stagnation of talks in multilateral system it will argue that the main consequence of a successful end of the current negotiation process would be the creation of Free Trade Area designed in the terms of US interest.

* Condell 249, Providencia, Santiago, Chile. Teléfono: (56) (2) 24961200. Correo electrónico: rodrigocuevas@uchile.cl. Investigador asociado Cátedra OMC/ Instituto de Estudios Internacionales, Universidad de Chile.
\end{abstract}


JEL classification: F13 - F15

Key words: regional trade agreements, negotiating process, trade blocs, free trade.

\section{INTRODUCCION}

El Trans-Pacific Partnership (TPP) es un proceso negociador que oficialmente involucra a 12 países (Australia, Brunei Darussalam, Canadá, Chile, Estados Unidos, Japón, Malasia, México, Nueva Zelanda, Perú, Singapur y Vietnam), a los cuales probablemente se les unirá Corea del Sur ${ }^{1}$. Representaría una novedad por cuanto es un proceso con un alto grado de cobertura geográfica (comprende a países de América, Asia y Oceanía) que involucra a países con una amplia diferencia en cuanto a sus niveles de ingresos.

Esta negociación ha generado importantes expectativas, al ser considerada una de las más importantes de los últimos años, al ser lo que se denominaría un Acuerdo del Siglo XXI. Entre sus principales características se mencionan una amplia flexibilidad en la incorporación de nuevos integrantes y una ambiciosa agenda que incluiría compromisos con alto grado de profundidad por parte de los países que lo suscriban ${ }^{2}$, tanto con respecto al ámbito de la OMC $(\mathrm{WTO}+)^{3}$, de lo logrado por otros Acuerdos Comerciales Regionales(ACR $)^{4}$, o incluso que profundicen aquellos aspectos que no están contenidos en la esfera multilateral (WTO-

1 En estricto rigor, en su análisis Schott, Kotschwar y Muir trabajan sobre dos escenarios posibles. Uno es con un escenario de 11 países negociadores (TPP11), puesto que Japón recién se integró formalmente en Julio de este año, y otro que considera tanto a Japón como a Corea del Sur (TPP13). Al respecto ver Schott, J., Kotschwar, B., \& Muir, J. (2013).

2 Lim. C. L, Elms, D. K, \& Low, P. (2012).

3 El concepto WTO Plus (WTO +) se refiere a aquellas disposiciones contenidas en Acuerdos Comerciales Regionales que profundizan el nivel de compromisos por lo dispuesto en el ámbito de la OMC, como por ejemplo el caso de la propiedad intelectual. Por su parte, el concepto WTO extra (WTO-x) se refiere a aquellas disposiciones presentes en ACR que van más allá que lo contemplado en la OMC. Al respecto ver Horn, H., Mavroidis, P., \& Sapir, A. (2009).

Dependiendo del tipo de acuerdo, la OMC los clasifica como "Unión Aduanera", "Acuerdo de Libre Comercio", “Acuerdo de Libre Comercio e Integración Económica”, "Acuerdo de Alcance Parcial" y "Acuerdo de Alcance Parcial e Integración Económica”. En algunos casos, un mismo tipo de acuerdo puede recibir nombres distintos. Este tipo de acuerdos recibe distintos tipos de nombres (ej.: Tratado de Libre Comercio, Acuerdo de Asociación, etc.). Tomando en cuenta estas consideraciones, resulta más útil para efectos de este trabajo utilizar la denominación "Acuerdos Comerciales Regionales", que es como los agrupa la propia OMC. Al respecto ver Regional Trade Agreements Information System (RTA-IS), World Trade Organization en http://rtais.wto.org/ui/ PublicMaintainRTAHome.aspx 
$\mathrm{X})$. Entre estas disposiciones que se incorporarían en el TPP esté la convergencia en las reglas de origen presentes en anteriores acuerdos, elevar los estándares de protección a la propiedad intelectual y a las inversiones, profundizar el comercio de servicios (negociación en lista negativa), entre otras ${ }^{5}$.

Si bien estas características hacen de esta negociación un objeto de gran interés, el análisis de este proceso no es sencillo. Pese a ser un caso sobre el cual hay trabajos académicos ${ }^{6}$, la falta de un ACR sobre el cual realizar una comparación hace que el análisis se construya sobre escenarios hipotéticos. A esta dificultad se añade que es un caso sobre el cual no abunda la información oficial, salvo la proporcionada por los gobiernos respectivos, medios periodísticos, como columnas publicadas por ex negociadores, tanto de éste como de acuerdos previos ${ }^{7}$. Además, las negociaciones del TPP no figuran dentro de los procesos de este tipo en curso que se han notificado como tales ante la Organización Mundial del Comercio $(\mathrm{OMC})^{8}$, pese a iniciarse con bastante visibilidad en marzo de 2010.

El objetivo del presente artículo es presentar el proceso de negociación de este Acuerdo del Siglo XXI, identificando intereses de los distintos países involucrados así como aquellos elementos que aparecerían como claves en las negociaciones. Asimismo, se señala la dimensión política de lo que representaría el TPP. El argumento principal es que si bien oficialmente se trata de 12 países negociando, este proceso en realidad sería uno de EE.UU. con otros 11, debido tanto a las grandes diferencias entre ese país y el resto de las partes, como a las características de la negociación, que se asemejan a los ACR firmados por ese país.

La estructura del presente documento es la siguiente. Primero se realiza una revisión de las principales motivaciones que llevan a los países a buscar este tipo de acuerdos, situando la negociación dentro de un contexto de un crecimiento

5 Una lista negativa es que toda apertura en inversión o servicio se da por sentada, a menos que se incluya explícitamente como una excepción; sólo se incluyen aquellos sectores que no estarán presentes, dándose por sentado que todo lo que no se incorpore explícitamente será parte del ACR. En general, las negociaciones en estas materias no utilizan este sistema, sino el de lista positiva, que utiliza la lógica inversa, de negociar sólo lo explícitamente indicado. Al respecto ver Goode, W. (2007).

6 A modo de referencia, dos completas publicaciones al respecto son las siguientes: Lim. C. L, Elms, D. K., \& Low, P. (Eds.). (2012) y Schott, J., Kotschwar, B., \& Muir, J. (2013).

7 Contreras, R. (2013). El Acuerdo de Asociación Transpacífico en la política comercial chilena. Revista Puentes, Volumen 14, No3, pp 4-8 y Rosales, O. Reflexiones sobre el TPP. 10 de Junio de 2013. Radio Cooperativa. Recuperado de www.cooperativa.cl

8 Regional Trade Agreements Information System (RTA-IS), OMC. http://rtais.wto.org/UI/ PublicMaintainRTAHome.aspx, información disponible al 18 de Julio de 2013. 
explosivo de ACR a nivel global, realizándose una síntesis de los principales intereses de los países presentes en las negociaciones para buscar incorporarse al TPP. En segundo lugar, se describen las negociaciones que se están llevando a cabo desde sus inicios hasta la $18^{\text {a }}$ Ronda, realizada en julio de 2013. En tercer lugar, se exponen la dimensión más bien política de las negociaciones del TPP. Finalmente, se presentan las conclusiones.

\section{I. ¿POR QUE SE NEGOCIA UN ACR?}

Las principales razones que llevan a los países a negociar este tipo de acuerdos son de tipo económico y político, donde ambas dimensiones suelen estar entrelazadas. Una aproximación útil es la que realiza Ravenhill, para quien las razones políticas serían las siguientes. Primero, la búsqueda de la cooperación económica y construcción de confianza. Segundo, la firma de acuerdos comerciales como un complemento de una política de seguridad. Tercero, concebir este tipo de acuerdos regionales como una forma de reducir asimetrías de poder frente a empresas transnacionales y a otros bloques regionales. Cuarto, la búsqueda, mediante un Acuerdo Internacional, de consolidar internamente procesos de reformas económicas estructurales. Quinto, los ACR como una herramienta mediante la cual satisfacer a una serie de sectores internos a través de la obtención de condiciones ventajosas de apertura que no serían posibles de conseguir mediante un acuerdo multilateral. Sexto, la opción de los ACR como una ventaja frente a negociaciones multilaterales en determinados períodos de estancamiento de las mismas. En este último punto, si bien no es antecedente concluyente, a partir del estancamiento en las negociaciones multilaterales en el marco de la Ronda de Doha ${ }^{9}$, especialmente desde 2003 tras el fracaso en la reunión ministerial de Cancún, el crecimiento en el número de ACR se acelera aún más (Gráfico 1). A ello se agregarían el papel de determinadas agencias gubernamentales/intergubernamentales que tendrían el poder de agregar intereses e influir en las respectivas agendas de negociación ${ }^{10}$.

En el caso de las motivaciones económicas, estarían las siguientes. En pri-

9 La denominación Ronda refiere a las negociaciones económicas de tipo multilateral. En el caso de Ronda de Doha (que debe su nombre a que fue lanzada en la capital de Qatar en 2001) es la primera que se realiza después de establecida la OMC, y es la continuación de aquellos temas que habían quedado inconclusos tras la finalización de la Ronda Uruguay (especialmente Agricultura) y que se había establecido que debían ser vueltos a negociar en el mediano plazo, tras la puesta en marcha de la OMC. Al respecto ver Gallagher, P. (2005).

10

Si bien se enfoca en un caso bastante poco comparable con otros, es muy interesante al respecto el artículo de Elsig sobre el papel de la Comisión Europea en la inclusión de temáticas regulatorias en los ACR en que participa la Unión Europea. Al respecto ver Elsig, M. (2007). 
mer lugar, proteger a sectores que no sobrevivirían a una apertura no concertada. Segundo, proporcionar oportunidades para una integración más profunda. Tercero, expandir mercados y atraer mayor inversión extranjera (Ravenhill, 2011). Junto a la clasificación de Ravenhill, es muy interesante para efectos de este artículo el concepto de Teoría del Efecto Dominó, de Richard Baldwin (2011), que plantea que determinados ACR tienen como consecuencia la búsqueda de nuevos acuerdos o la incorporación a los previamente firmados por el costo que significaría ser excluido de los beneficios contemplados a quienes sí los integran. Ello explicaría, por ejemplo, el caso de países latinoamericanos, especialmente Chile, tras la firma del NAFTA (Carpenter, 2008).

Más recientemente destaca el estudio realizado por Baccini, Dür, Elsig y Milewicz (2011), quienes a partir de la codificación de ACR y 10 de sus más importantes temàticas ${ }^{11}$, construyen una nueva base de acuerdos (Design of Trade Agreements, DESTA), que amplía a otras existentes, como de la OMC o la del Banco Mundial $^{12}$. En DESTA trabajan sobre un total de 440 ACR (sobre un potencial de 690) firmados entre 1945 y 2009. Como tendencia, identifican a partir de 1990 un incremento sustantivo de acuerdos entre países con distinto nivel de desarrollo, donde primaría la presencia de mayores niveles de profundización en materia de protección a las inversiones y a la propiedad intelectual, especialmente cuando uno de sus integrantes, uno al menos, sea un país altamente industrializado.

Los ACR se definen como preferenciales, por cuanto permiten hacer una excepción a la cláusula de Nación más Favorecida (NMF), pudiendo restringir los beneficios sólo a sus integrantes sin la necesidad de extenderlos a terceros ${ }^{13}$, constituyéndose de esta manera en una vía paralela al Sistema Multilateral de Comercio (SMC) permitida por la propia OMC, proporcionando un alto grado de autonomía de los países al permitirles eximirse, constituyéndose la política comercial un instrumento en el cual canalizan e implementan objetivos e intereses. $\mathrm{Si}$

${ }^{11}$ Los autores incluyen, entre otras, acceso a mercados, servicios, derechos de propiedad intelectual, contratación pública, política de competencia, inversiones y medidas de defensa comercial. Ibid

12 Global Preferential Trade Agreements Database, World Bank, http://wits.worldbank.org/gptad/

13 Se trata principalmente de los Artículos XXIV del Acuerdo GATT 1994, y del Artículo V del ACGS. Al respecto ver OMC, (2003) Los textos jurídicos. Los resultados de la Ronda Uruguay de Negociaciones Comerciales Multilaterales, op-cit. Al mismo tiempo, al revisar la base de datos de ACR de la OMC, una gran mayoría de los acuerdos de este tipo firmados se basan en la invocación de alguno de estos o ambos artículos. Otro motivo, que se ve especialmente en acuerdos anteriores a 1995, es la clausula de habilitación, que era un mecanismo previsto en la Ronda de Tokio, en el marco del GATT, que preveía la creación de ACR entre países en desarrollo. Al respecto, ver RTAIS, OMC. Op-cit. 
bien el uso de los ACR ha despertado importantes críticas, particularmente porque su uso constituiría un gran daño al propio $\mathrm{SMC}$, en la práctica los países continúan y probablemente sigan buscando este tipo de acuerdos (Bhagwatti, 2008).

$\mathrm{Al}$ analizar las negociaciones del TPP, es necesario contextualizarlas dentro del acelerado crecimiento de los ACR desde 1990 a 2013, período en el cual se concentran 236 acuerdos en vigor ${ }^{14}$, que representan un $90 \%$ de la totalidad de acuerdos del periodo que va desde 1958 a julio de 2013 (Gráfico 1). Pese a que, dentro del período iniciado en el contexto de las negociaciones del Acuerdo General de Comercio y Aranceles (GATT), en 1947 se da el uso de este tipo de instrumentos (el primer acuerdo registrado en la $\mathrm{OMC}$ es el de las Comunidades Europeas, CE), los 25 acuerdos en vigor antes de 1990 correspondieron en su mayoría tanto a procesos de ampliación de la CE (1973, 1981 y 1986) como acuerdos de ésta con otros países europeos o ex territorios coloniales ${ }^{15}$.

Gráfico 1 - ACR’s en vigor, 1958-2013

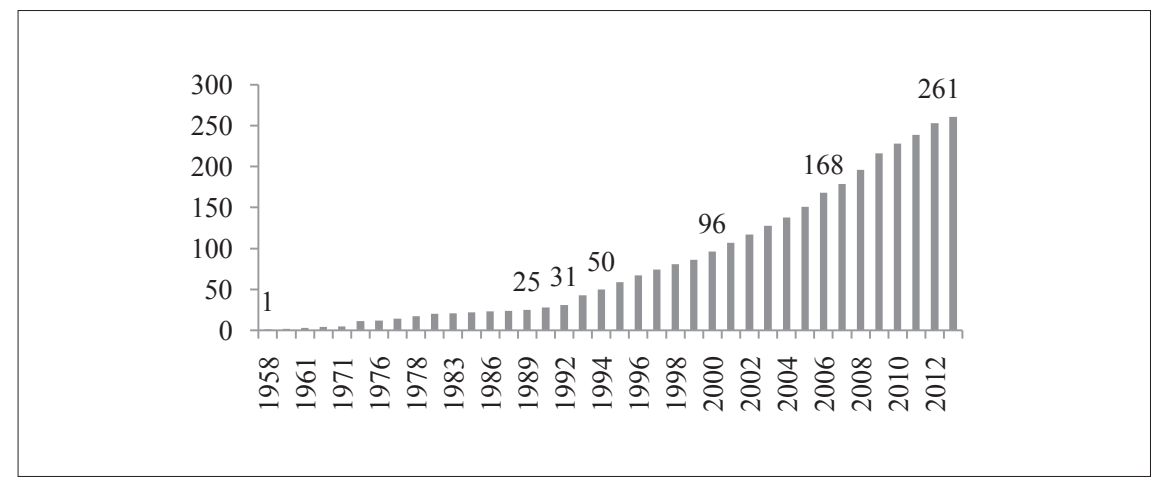

Fuente: Regional Trade Agreements Information System (RTA-IS), OMC.

http://rtais.wto.org/UI/PublicMaintainRTAHome.aspx, Julio de 2013.

Los 12 países que serían eventualmente parte del TPP cuentan con un total de 151 acuerdos, de los cuales un 70\% (106) se hallan en vigor y un 23\% (35) se encuentran en negociaciones. Un número marginal se encuentra firmado y con-

14 Los datos entregados respecto a los ACR son tomados a partir de la base de datos de la OMC sobre ACR, que se denomina Regional Trade Agreements Information System (RTA-IS). Al respecto, ver OMC. http://rtais.wto.org/UI/PublicMaintainRTAHome.aspx.

15 Asimismo, se cuentan algunos acuerdos regionales en Oceanía o ACR plurilaterales en América Latina, como el Pacto Andino, el Mercado Común Centroamericano o la Asociación Latinoamericana de Integración (ALADI). 
cluido, $5 \%$ (7) y $2 \%$ (3), respectivamente. Si se toma en consideración los que se encuentran en vigor, un 27\% (29) de los ACR corresponden a países que negocian el TPP (Cuadro 1).

Cuadro 1: ACR de países negociadores, según estado

\begin{tabular}{cccccc}
\hline & Vigor & Firmado & Concluida & $\begin{array}{c}\text { En } \\
\text { negociación }\end{array}$ & Total \\
\hline $\begin{array}{c}\text { ACR con miembro } \\
\text { del TPP }\end{array}$ & 29 & 1 & 1 & 6 & 37 \\
$\begin{array}{c}\text { ACR con no } \\
\text { miembro del TPP }\end{array}$ & 77 & 6 & 2 & 29 & 114 \\
Total & 106 & 7 & 3 & 35 & 151 \\
\hline
\end{tabular}

* Sin incluir al TPP. Información recopilada a Octubre de 2013.

Fuente: Regional Trade Agreements Information System (RTA-IS), OMC. y gobiernos de países TPP

Al agrupar el listado de acuerdo a cada integrante del TPP (Cuadro 2), los resultados muestran que Chile, Singapur y México son los países que más han utilizado la vía de los ACR en sus procesos de apertura comercial. En el caso de los dos primeros, junto con EE.UU., son los países que más tienen o negocian algún acuerdo con algún integrante del potencial Acuerdo del Siglo XXI. Al mismo tiempo, los doce países integrantes se encuentran en conversaciones en 35 acuerdos, donde Canadá aparece como el país más activo, con 9 acuerdos enfocados en su mayoría en América Latina, aunque con países como Japón y bloques como la UE. Si bien EE.UU. aparece con sólo un ACR en negociación, se trata de una contraparte muy significativa, como es la $\mathrm{UE}^{16}$.

Cuadro 2: ACR de países negociadores del TPP, según estado*

16 Esta negociación fue notificada formalmente a la OMC el 20 de Julio de 2013. Ver Regional Trade Agreements Information System(RTA-IS), OMC. http://rtais.wto.org/UI/PublicMaintainRTAHome. aspx 


\begin{tabular}{|c|c|c|c|c|c|c|c|c|c|c|}
\hline & ACR & $\begin{array}{l}\text { ACR con } \\
\text { miembros } \\
\text { del TPP }\end{array}$ & & & & & & & & \\
\hline & V & $\mathrm{F}$ & $\mathrm{C}$ & $\mathrm{N}$ & Total & V & $\mathrm{F}$ & $\mathrm{C}$ & $\mathrm{N}$ & Total \\
\hline Australia & 8 & -- & -- & 8 & 16 & 6 & -- & -- & 2 & 8 \\
\hline $\begin{array}{c}\text { Brunei } \\
\text { Darussalam }\end{array}$ & 8 & -- & -- & 1 & 9 & 5 & -- & -- & 1 & 6 \\
\hline Canadá & 11 & -- & 1 & 9 & 21 & 3 & -- & -- & 2 & 5 \\
\hline Chile & 27 & 2 & 1 & 1 & 31 & 8 & 1 & -- & 1 & 10 \\
\hline EE.UU. & 14 & -- & -- & 1 & 15 & 5 & -- & -- & -- & 10 \\
\hline Japón & 13 & -- & -- & 5 & 18 & 8 & -- & -- & 3 & 11 \\
\hline Malasia & 14 & 2 & -- & 3 & 19 & 8 & -- & -- & 1 & 9 \\
\hline México & 20 & -- & -- & 3 & 23 & 4 & -- & -- & 2 & 6 \\
\hline $\begin{array}{c}\text { Nueva } \\
\text { Zelandia }\end{array}$ & 6 & -- & 1 & 4 & 11 & 4 & -- & 1 & 1 & 6 \\
\hline Perú & 8 & 1 & -- & 3 & 13 & 5 & -- & -- & 1 & 6 \\
\hline Singapur & 18 & 1 & -- & 5 & 24 & 7 & -- & -- & 3 & 10 \\
\hline Vietnam & 8 & 1 & -- & 2 & 11 & 4 & 1 & -- & 1 & 6 \\
\hline
\end{tabular}

* Sin considerar el TPP. Información recopilada a Octubre de 2013.

Vigor (V). Firmado (F) Negociación Concluida (C) En Negociaciones (N)

Fuente: Regional Trade Agreements Information System (RTA-IS), OMC. y gobiernos de países TPP

Analizar los resultados por país da cuenta de una situación bastante significativa, que es el uso intensivo de los ACR por parte de los miembros del TPP, puesto que la mayoría de ellos ya cuenta con acuerdos previos entre sí, y en algunos casos con prácticamente todos los países integrantes (Cuadro 3). Ello muestra que la negociación bilateral es una vía para nada descartada por la mayoría de los países negociadores, siendo Canadá y Japón los miembros más activos en el uso de este camino, seguidos de Australia, Singapur y México. Incluso Vietnam, que es el país que menos ACR tiene, firmó entre 2010 y 2013 uno en paralelo con Chile (Cuadro 3). 
TRANS-PACIFIC-PARTNERSHIP: EL COMPLEJO CAMINO DEL ACUERDO DEL SIGLO XX

Cuadro 3: Acuerdos bilaterales entre países negociadores del TPP ${ }^{17}$

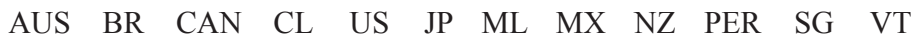

\begin{tabular}{|c|c|c|c|c|c|c|c|c|c|c|c|c|}
\hline $\begin{array}{l}\text { Australia } \\
\text { (AUS) }\end{array}$ & & $\mathrm{V}^{1}$ & $\mathrm{P}$ & V & $\mathrm{V}$ & $\mathrm{N}$ & V & $\mathrm{P}$ & V & $\mathrm{P}$ & $\mathrm{V}$ & $\mathrm{V}^{1}$ \\
\hline $\begin{array}{c}\text { Brunei } \\
\text { Darussalam } \\
(\mathrm{BR})\end{array}$ & $\mathrm{V}^{1}$ & & $\mathrm{P}$ & $\mathrm{V}^{3}$ & $\mathrm{P}$ & V & $\mathrm{V}^{2}$ & $\mathrm{P}$ & $\mathrm{V}^{3}$ & $\mathrm{P}$ & $\mathrm{V}^{3}$ & $\mathrm{~V}^{2}$ \\
\hline $\begin{array}{l}\text { Canadá } \\
\text { CAN) }\end{array}$ & $\mathrm{P}$ & $\mathrm{P}$ & & V & $\mathrm{V}^{4}$ & $\mathrm{~N}$ & $\mathrm{P}$ & $\mathrm{V}^{4}$ & $\mathrm{P}$ & V & $\mathrm{N}$ & $\mathrm{P}$ \\
\hline Chile (CL) & $\mathrm{V}$ & $\mathrm{V}^{3}$ & $\mathrm{~V}$ & & $\mathrm{~V}$ & V & V & $\mathrm{V}$ & $\mathrm{V}^{3}$ & V & $\mathrm{V}^{3}$ & $\mathrm{~F}$ \\
\hline $\begin{array}{c}\text { EE.UU. } \\
\text { (US) }\end{array}$ & $\mathrm{V}$ & $\mathrm{P}$ & $\mathrm{V}^{4}$ & $\mathrm{~V}$ & & $\mathrm{P}$ & $\mathrm{P}$ & $\mathrm{V}^{4}$ & $\mathrm{P}$ & V & $\mathrm{V}$ & $\mathrm{P}$ \\
\hline Japón (JP) & $\mathrm{N}$ & $\mathrm{V}$ & $\mathrm{N}$ & V & $\mathrm{P}$ & & $\mathrm{V}$ & $\mathrm{V}$ & $\mathrm{P}$ & V & $\mathrm{V}$ & $\mathrm{V}$ \\
\hline $\begin{array}{l}\text { Malasia } \\
(\mathrm{ML})\end{array}$ & $\mathrm{V}$ & $\mathrm{V}^{2}$ & $\mathrm{P}$ & $\mathrm{V}$ & $\mathrm{P}$ & $\mathrm{V}$ & & $\mathrm{P}$ & $\mathrm{V}$ & $\mathrm{P}$ & $\mathrm{V}^{2}$ & $\mathrm{~V}^{2}$ \\
\hline $\begin{array}{l}\text { México } \\
(\mathrm{MX})\end{array}$ & $P$ & $\mathrm{P}$ & $\mathrm{V}^{4}$ & $\mathrm{~V}$ & $\mathrm{~V}^{4}$ & V & V & & $\mathrm{P}$ & V & $\mathrm{N}$ & $\mathrm{P}$ \\
\hline $\begin{array}{c}\text { Nueva } \\
\text { Zelanda (NZ) }\end{array}$ & $\mathrm{V}$ & $\mathrm{V}^{3}$ & $\mathrm{P}$ & $\mathrm{V}^{3}$ & $\mathrm{P}$ & $\mathrm{P}$ & $\mathrm{P}$ & $\mathrm{P}$ & & $\mathrm{P}$ & $\mathrm{V}^{3}$ & $\mathrm{~V}^{1}$ \\
\hline Perú (PER) & $\mathrm{P}$ & $\mathrm{P}$ & $\mathrm{V}$ & $\mathrm{V}$ & $\mathrm{V}$ & V & $\mathrm{P}$ & $\mathrm{V}$ & $\mathrm{P}$ & & $\mathrm{V}$ & $\mathrm{P}$ \\
\hline $\begin{array}{l}\text { Singapur } \\
\text { (SG) }\end{array}$ & $\mathrm{V}$ & $\mathrm{V}^{3}$ & $\mathrm{~N}$ & $\mathrm{~V}^{3}$ & $\mathrm{~V}$ & V & $\mathrm{V}^{2}$ & $\mathrm{~N}$ & $\mathrm{~V}^{3}$ & V & & $\mathrm{V}^{2}$ \\
\hline $\begin{array}{l}\text { Vietnam } \\
\text { (VT) }\end{array}$ & $\mathrm{V}^{1}$ & $\mathrm{~V}^{2}$ & $\mathrm{P}$ & $\mathrm{F}$ & $\mathrm{P}$ & $\mathrm{V}$ & $\mathrm{V}^{2}$ & $\mathrm{P}$ & $\mathrm{V}^{1}$ & $\mathrm{P}$ & $\mathrm{V}^{2}$ & \\
\hline
\end{tabular}

Vigor (V). Firmado (F) Negociación (N) Posible Acuerdo en TPP (P) ${ }^{1}$ Acuerdos firmados con ASEAN.

${ }^{2}$ Miembros de ASEAN. ${ }^{3}$ P-4. ${ }^{4}$ NAFTA.

Fuente: Regional Trade Agreements Information System (RTA-IS), OMC. y gobiernos de países TPP.

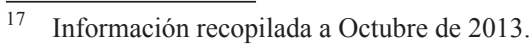


La presencia de una importante red de acuerdos previos explicaría en parte el interés por negociar un acuerdo de las características del TPP, por el atractivo de buscar un acuerdo que regule la presencia de reglas de origen, debido a que deberían converger las normas que fueron pensadas para $37 \mathrm{ACR}$ distintos ${ }^{18}$, debido a que este tipo de normas busca principalmente asegurar que los beneficios contemplados correspondan exclusivamente a bienes producidos en algunas de las partes de un acuerdo y que se apliquen a una eventual tercera parte que tenga algún ACR distinto al contemplado para una respectiva regla de origen (Liang, 2012). En éstos coexisten desde enfoques más abiertos, como el de los países asiáticos que tienen reglas de origen más flexibles que permiten la acumulación (la presencia de terceros países ajenas al ACR en cuestión), frente a regímenes más restrictivos, como es el caso de los ACR de EE.UU ${ }^{19}$. Al revisar este, ello resulta de gran importancia en países no desarrollados y que son exportadores relevantes de manufacturas, como es el caso de países asiáticos (con la excepción de Brunei Darussalam) y México, quienes con reglas de origen flexibles podrían incorporarse de una manera más eficaz en un patrón de comercio que tiene una importante característica la integración de la cadena de valor a nivel global, proceso que la OMC lo explica bajo el concepto Made in the World ${ }^{20}$.

Al considerar las cifras globales del comercio entre los 12 países presentes en las negociaciones (Cuadro 4), destaca la gran importancia que representaría el TPP para Canadá y México, con un 71\% y $69 \%$ del total de sus exportaciones e importaciones de bienes respectivamente, aunque en realidad el porcentaje se explica por una situación muy específica, que es la importancia de EE.UU. para ambos tras el NAFTA, por lo que es una situación que no representa un gran cambio. También destaca el caso de Brunei Darussalam, para el que este potencial acuerdo representa un $62 \%$ de su comercio, cifra en la que casi la mitad corresponde a Japón, país con el que ya cuenta con un ACR en vigor. Para el resto de los países, en general el TPP representa una proporción importante en sus flujos comerciales, con porcentajes entre el $22 \%$ de Vietnam y el 39\% de EE.UU. Un segundo elemento a considerar es la importancia del comercio intrarregional, tanto en América del Norte como entre países del Asia-Pacífico, relevancia que disminuye en el caso de los países latinoamericanos.

18 Sumados Acuerdos entre en vigor, firmados, concluidos y en negociación.

19 Ibid.

20 Al respecto, ver en el sitio web de la OMC, http://www.wto.org/english/res_e/statis_e/miwi_e/ miwi_e.htm. Una publicación reciente respecto a ese tema es Elms, D. K., \& Low, P. (Eds.). (2013). 
TRANS-PACIFIC-PARTNERSHIP: EL COMPLEJO CAMINO DEL ACUERDO DEL SIGLO XX

Cuadro 4: Comercio de bienes entre países del TPP, 2012 (\%)

\begin{tabular}{|c|c|c|c|c|c|c|c|c|c|c|c|c|}
\hline & AUS & BR & CAN & CL & US & $\mathrm{JP}$ & ML & MX & $\mathrm{NZ}$ & PER & $\mathrm{SG}$ & VT \\
\hline AUS & & 6,2 & 0,4 & 1,1 & 1,0 & 4,4 & 3,3 & 0,3 & 17,8 & 0,3 & 2,8 & 2,3 \\
\hline BR & 0,2 & & 0,0 & 0,0 & 0,0 & 0,4 & 0,2 & 0,0 & 1,3 & 0,0 & 0,2 & 0,1 \\
\hline CAN & 0,8 & 0,1 & & 1,5 & 15,5 & 1,4 & 0,4 & 2,8 & 1,2 & 5,7 & 0,3 & 0,6 \\
\hline CL & 0,3 & 0,0 & 0,3 & & 1 & 1 & 0 & 1 & 0 & 4 & 0 & 0 \\
\hline US & 7,7 & 2,3 & 62,2 & 17,6 & & 13 & 8 & 64 & 9 & 16 & 8 & 11 \\
\hline $\mathrm{JP}$ & 13,7 & 36,2 & 2,9 & 7,0 & 5,8 & & 0 & 0 & 0 & 0 & 0 & 0 \\
\hline ML & 3,0 & 4,8 & 0,3 & 0,3 & 1,0 & 3,0 & & 0,7 & 2,9 & 0,3 & 11,5 & 3,3 \\
\hline MX & 0,6 & 0,0 & 3,4 & 2,5 & 12,3 & 0,9 & 0,4 & & 1 & 2 & 0 & 0 \\
\hline $\mathrm{NZ}$ & 3,0 & 4,0 & 0,1 & 0,1 & 0,2 & 0,3 & 0,5 & 0,1 & & 0 & 0 & 0 \\
\hline PER & 0,0 & 1,4 & 0,5 & 2,5 & 0,4 & 0,2 & 0,0 & 0,3 & 0,2 & & 0 & 0 \\
\hline SG & 4,4 & 6,5 & 0,2 & 0,1 & 1,3 & 1,9 & 13,4 & 0,3 & 3,2 & 0,1 & & 4 \\
\hline VT & 1,0 & 3,6 & 0,2 & 0,4 & 0,7 & 1,5 & 2,1 & 0,2 & 0,8 & 0,2 & 1,6 & \\
\hline $\mathrm{TPP}^{1}$ & 34,9 & 65,2 & 70,5 & 32,9 & 39 & 28 & 29 & 69 & 38 & 29 & 25 & 22 \\
\hline $\mathrm{TM}$ & 100 & 100 & 100 & 100 & 100 & 100 & 100 & 100 & 100 & 100 & 100 & 100 \\
\hline
\end{tabular}

*Los porcentajes de Perú y Vietnam corresponden a 2011.

Fuente: World Integrated Trade Solution (WITS), Banco Mundial. 
Cuadro 5: Exportaciones de bienes hacia países del TPP, 2012 (\%)

\begin{tabular}{|c|c|c|c|c|c|c|c|c|c|c|c|c|}
\hline & AUS & $\mathrm{BR}$ & CAN & CL & US & JP & ML & MX & $\mathrm{NZ}$ & PER & $\mathrm{SG}$ & VT \\
\hline AUS & & 0,3 & 0,4 & 1,6 & 2,1 & 2,3 & 4,1 & 0,3 & 20,5 & 0,3 & 4,2 & 2,7 \\
\hline BR & 0,0 & & 0,0 & 0,0 & 0,0 & 0,0 & 0,3 & 0,0 & 0,0 & 0,0 & 0,4 & 0,0 \\
\hline CAN & 0,7 & 0,0 & & 1,6 & 18,0 & 1,3 & 0,4 & 2,9 & 1,2 & 9,2 & 0,3 & 1,0 \\
\hline CL & 0,2 & 0,0 & 0,2 & & 1,3 & 0,2 & 0,1 & 0,6 & 0,2 & 4,3 & 0,0 & 0,1 \\
\hline US & 3,7 & 0,7 & 74,2 & 12,3 & & 17,8 & 8,7 & 77,7 & 9,2 & 13,3 & 5,5 & 17,5 \\
\hline JP & 19,4 & 44,1 & 2,4 & 10,7 & 4,8 & & 11,8 & 0,7 & 7,2 & 4,8 & 4,4 & 11,4 \\
\hline ML & 2,1 & 0,7 & 0,2 & 0,3 & 0,8 & 2,2 & & 0,1 & 2,0 & 0,0 & 12,3 & 2,9 \\
\hline MX & 0,4 & 0,0 & 1,1 & 1,7 & 12,9 & 1,3 & 0,7 & & 0,6 & 1,0 & 0,3 & 0,6 \\
\hline $\mathrm{NZ}$ & 3,0 & 5,1 & 0,1 & 0,1 & 0,2 & 0,2 & 0,5 & 0,0 & & 0,0 & 0,5 & 0,2 \\
\hline PER & 0,1 & 0,0 & 0,1 & 2,3 & 0,6 & 0,1 & 0,1 & 0,4 & 0,3 & & 0,0 & 0,1 \\
\hline SG & 2,9 & 1,8 & 0,2 & 0,1 & 2,0 & 2,9 & 13,6 & 0,2 & 1,8 & 0,0 & & 2,2 \\
\hline VT & 0,7 & 4,6 & 0,1 & 0,5 & 0,3 & 1,3 & 1,7 & 0,0 & 1,0 & 0,2 & 2,5 & \\
\hline ТPP & 33,0 & 57,3 & 79,0 & 31,2 & 43,1 & 29,8 & 41,9 & 83,0 & 44,0 & 33,1 & 30,5 & 38,7 \\
\hline TM & 100 & 100 & 100 & 100 & 100 & 100 & 100 & 100 & 100 & 100 & 100 & 100 \\
\hline
\end{tabular}

*Los porcentajes de Perú y Vietnam corresponden a 2011.

Fuente: World Integrated Trade Solution (WITS), Banco Mundial.

Al desglosar las cifras, según las exportaciones, se repite un cuadro similar a los flujos comerciales globales acentuándose en el caso de Canadá y México. Sin embargo, es interesante ver el caso de Nueva Zelanda, donde en el TPP aparece como un importante destino de sus envíos, $44 \%$, aunque casi la mitad de ellos se explica por la importancia de Australia, con un 21\% (Cuadro 5). En el caso de las importaciones, destaca el caso de Brunei Darussalam, que importa el 90\% desde países del TPP, que se explica principalmente por la importancia de Australia, Sin- 
gapur y Malasia. También es relevante el caso de Canadá, el que nuevamente se explica por su alta vinculación con EE.UU. a partir del NAFTA (Cuadro 6).

Cuadro 6: Importaciones de bienes de países del TPP, 2012 (\%)

\begin{tabular}{|c|c|c|c|c|c|c|c|c|c|c|c|c|}
\hline & AUS & $\mathrm{BR}$ & CAN & $\mathrm{CL}$ & US & JP & ML & MX & $\mathrm{NZ}$ & PER & SG & VT \\
\hline AUS & & 30,6 & 0,5 & 0,6 & 0,4 & 6,4 & 2,4 & 0,3 & 15,3 & 0,3 & 1,3 & 2,0 \\
\hline BR & 0,0 & & 0,0 & 0,0 & 0,0 & 0,7 & 0,0 & 0,0 & 2,6 & 0,0 & 0,0 & 0,2 \\
\hline CAN & 1,0 & 0,3 & & 1,3 & 14,0 & 1,4 & 0,5 & 2,7 & 1,2 & 1,5 & 0,3 & 0,3 \\
\hline $\mathrm{CL}$ & 0,5 & 0,0 & 0,4 & & 0,4 & 1,1 & 0,1 & 0,4 & 0,1 & 3,5 & 0,0 & 0,3 \\
\hline US & 11,8 & 7,8 & 51,0 & 22,9 & & 8,8 & 8,1 & 50,1 & 9,4 & 19,7 & 10,2 & 4,3 \\
\hline $\mathrm{JP}$ & 7,9 & 7,5 & 3,3 & 3,3 & 6,4 & & 10,3 & 4,8 & 6,5 & 3,5 & 6,2 & 9,7 \\
\hline ML & 4,0 & 19,9 & 0,5 & 0,3 & 1,1 & 3,7 & & 1,3 & 3,9 & 0,5 & 10,6 & 3,7 \\
\hline MX & 0,8 & 0,2 & 5,6 & 3,3 & 12,0 & 0,5 & 0,1 & & 0,5 & 3,7 & 0,4 & 0,1 \\
\hline $\mathrm{NZ}$ & 3,0 & 0,3 & 0,1 & 0,1 & 0,2 & 0,3 & 0,4 & 0,1 & & 0,2 & 0,2 & 0,4 \\
\hline PER & 0,0 & 0,0 & 0,8 & 2,6 & 0,3 & 0,3 & 0,0 & 0,1 & 0,1 & & 0,0 & 0,1 \\
\hline SG & 6,0 & 23,5 & 0,3 & 0,1 & 0,9 & 1,0 & 13,2 & 0,4 & 4,5 & 0,1 & & 6,0 \\
\hline VT & 1,3 & 0,3 & 0,4 & 0,3 & 0,9 & 1,7 & 2,7 & 0,3 & 0,7 & 0,3 & 0,6 & \\
\hline ТPP & 36,3 & 90,4 & 62,7 & 34,7 & 36,7 & 25,9 & 37,8 & 60,3 & 44,7 & 33,3 & 30,0 & 27,0 \\
\hline TM & 100 & 100 & 100 & 100 & 100 & 100 & 100 & 100 & 100 & 100 & 100 & 100 \\
\hline
\end{tabular}

*Los porcentajes de Perú y Vietnam corresponden a 2011.

Fuente: World Integrated Trade Solution (WITS), Banco Mundial.

Sin embargo, ni la información señalada por los flujos comerciales entre los países del TPP ni la red de ACR's previos explican por sí solos las razones de los países por buscar este acuerdo. Un antecedente importante a considerar es el Trans-Pacific Strategic Economic Partnership (P-4), un acuerdo entre Brunei Da- 
russalam, Chile y Nueva Zelanda, que ayudó a impulsar acuerdos comerciales en la región; más de la mitad de los ACR que involucran a los países negociadores del TPP entre sí han entrado en vigor después de ese acuerdo ${ }^{21}$, por lo que habría un efecto del tipo "Teoría de Dominó". Este acuerdo, además, fue la base en la que se gestó el actual proceso negociador, que originalmente comenzó como un proceso de ampliación del P-4.

Para explicar las motivaciones de los países por ingresar es necesario tomar en cuenta los planteamientos de Ravenhill para explicar por qué los países buscan un Acuerdo del Siglo XXI. Al examinar la información proporcionada por los distintos gobiernos involucrados, muestran que el principal es el de un ACR con EE.UU., que aparecería como un motivo bastante evidente, porque la expectativa de un acceso preferencial al mercado de la principal economía del mundo es un objetivo sin dudas atractivo, y aparece explícito en los casos de Nueva Zelanda, Malasia y Vietnam (Cuadro 6). Sin embargo, ello no necesariamente explica el interés de todos los integrantes, pues seis de ellos ya cuentan un acuerdo con ese país $^{22}$. En ese sentido, es interesante el caso mexicano, pues si bien no busca el acceso al mercado estadounidense (ya es miembro del NAFTA ${ }^{23}$ ), el principal objetivo sería integrarse en la cadena de valor entre EE.UU. y Asia-Pacífico. Un segundo interés manifiesto, es el de la creación de un área de libre comercio, proceso en el que el TPP es visto como un gran paso. Un tercer argumento que aparece son las características de un eventual acuerdo (Siglo XXI) (Cuadro 7).

21 De los 29 acuerdos en vigor entre los 12 países, 14 entraron en vigor antes del P-4, mientras que 15 de los mismos lo hicieron de manera posterior. (RTA-IS), OMC, op.cit. y gobiernos de países negociadores. Dos de los cuatro integrantes del P-4 ya tenían un ACR con EE.UU. (Singapur), mientras que Australia tenía uno ya en vigor junto con Canadá y México, ambos miembros del NAFTA. Cabe señalar a Perú, que si bien su acuerdo con EE.UU. no estaba aún en vigor (entró en vigor en 2009), sí ya había sido firmado.

23 Siglas en ingles del Tratado de Libre Comercio de América del Norte, vigente entre Canadá, EE.UU. y México. 
Cuadro 7: Síntesis razones para ingresar al TPP

\begin{tabular}{lllll}
\hline & $\begin{array}{l}\text { ACR con } \\
\text { EE.UU. }\end{array}$ & $\begin{array}{l}\text { ALC }^{2} \text { en } \\
\text { APEC }\end{array}$ & ACR Siglo XXI & Otro \\
\hline Australia & & $\mathrm{X}$ & $\mathrm{X}$ & \\
Brunei Darussalam & & & $\mathrm{X}$ & \\
Canadá & & $\mathrm{X}$ & $\mathrm{X}$ & $\mathrm{X}^{3}$ \\
Chile & & $\mathrm{X}$ & $\mathrm{X}$ & $\mathrm{X}^{4}$ \\
EE.UU. & $\mathrm{X}$ & & $\mathrm{X}^{5}$ \\
Japón & $\mathrm{X}$ & $\mathrm{X}$ & $\mathrm{X}$ & $\mathrm{X}^{6}$ \\
Malasia & & $\mathrm{X}$ & & \\
México & $\mathrm{X}$ & $\mathrm{X}$ & $\mathrm{X}$ & $\mathrm{X}^{7}$ \\
Nueva Zelanda & & $\mathrm{X}$ & $\mathrm{X}$ & \\
Perú & & $\mathrm{X}$ & \\
Singapur & $\mathrm{X}$ & & & \\
Vietnam & & &
\end{tabular}

Fuente: Gobiernos de países negociadores.

\section{EL TPP: ANTECEDENTES, ESTADO DE LAS NEGOCIACIONES Y ESCENARIOS POSIBLES}

Originalmente, el TPP fue la búsqueda de la ampliación del P-4 hacia Estados Unidos, y buscaba entre sus objetivos la creación de un área de libre comercio en el marco del Foro Económico de Asia-Pacífico (APEC) (Wilhelmy, 2007). A diferencia de anteriores ACR's, el P-4 presenta una diferencia sustancial con otros acuerdos porque incorpora explícitamente la ampliación hacia nuevos integran$\operatorname{tes}^{24}$, siempre y cuando pertenezcan a $\mathrm{APEC}^{25}$, además de posibilitar a las partes asumir niveles diferenciados en la profundidad en sus compromisos (Wilhelmy, 2007). Sin embargo, lo que sería originalmente como un proceso de ampliación de este acuerdo, en realidad pasó a ser un potencial nuevo acuerdo en el marco de

\footnotetext{
24 Lewis, M. K. (2011).

25 Artículo 20.6 de Acuerdo Transpacífico de Asociación Económica, disponible en www.direcon.cl.
} 
$\mathrm{APEC}^{26}$, cuyo carácter fue principalmente dado por la incorporación de EE.UU. a las negociaciones por ser integrante del P-4. La entrada de este país, manifestada de manera en febrero de 2008, y oficializada en septiembre de ese año, trajo consigo de manera casi inmediata el interés de nuevos integrantes por participar, como es el caso de Australia, Perú y Vietnam, quienes manifestaron su intención en noviembre de ese año. Posteriormente, se agregaría en 2010 Malasia, Canadá y México, a fines de 2012, y Japón, en julio de $2013^{27}$.

Las negociaciones se iniciaron en marzo de 2010, con la presencia de ocho países: Australia, Brunei Darussalam, Chile, Estados Unidos, Nueva Zelanda, Perú, Singapur y Vietnam, realizándose a la fecha 17 rondas de negociaciones ${ }^{28}$. En la primera ronda negociadora de Melbourne, se dieron las primeras directrices respecto a la forma de este acuerdo, enfatizando la búsqueda de la inclusión de la mayor amplitud de temas posibles ${ }^{29}$. En la segunda ronda (San Francisco), se trazaron dos metas principales: el acceso a mercados y la relación con los acuerdos previamente existentes. En la tercera ronda (Brunei) se plantearon objetivos en la línea de mejorar la relación de los acuerdos ya vigentes y su aplicación en el ámbito interno (coherencia regulatoria) y en cómo mejorar la cadena de valor, que tiene relación con cómo las empresas pueden integrar estos acuerdos en sus procesos productivos, especialmente en lo relacionado con bienes intermedios. Estos aspectos se presentaron también durante la cuarta ronda.

En 2011, durante la quinta ronda (Santiago), se explicitaron públicamente, a través de representantes no gubernamentales, temas relacionadas con la propiedad intelectual y cómo ésta afectaría la libertad de expresión en Internet. En la

26 Lewis, M. K.(2011)

27 "Statement on the 18th Round of Trans-Pacific Partnership Negotiations", Office of the United States Representative, www.ustr.gov/tpp

28 El calendario de negociaciones ha sido el siguiente: en el año 2010 se realizaron cuatro rondas: Melbourne (15-19 de Marzo); San Francisco (14-18 de Junio); Brunei (4-9 de Octubre); Auckland (6-10 de Diciembre). En el 2011 se efectuaron rondas en Santiago (14-18 de Febrero), Singapur (24 Marzo - 1 Abril): Ho-Chi Minh (15-24 de Junio), Chicago (11-15 de Septiembre), Lima (22-29 de Octubre), Kuala Lumpur (5-9 de Diciembre). En 2012 se realizaron rondas en Melbourne (2-9 de Marzo), Dallas, (8-18 Mayo), San Diego (2-10 de Julio), Leesburg, Virginia (6-15 de Septiembre), Auckland (3-12 de diciembre). En 2013 se ha llevado a cabo una ronda negociadora en Singapur (413 de marzo) y Lima (15-24 de Mayo). Office of the United States Trade Representative (USTR). www.ustr.gov/tpp

29 La información sobre las Rondas de Negociación se tomó en base a los antecedentes recogidos de fuentes oficiales de los países negociadores. En ese sentido, el grueso de la misma se tomó en base a lo publicado por la Oficina del Representante de Comercio de Estados Unidos (USTR), que contenía la información más completa respecto a las negociaciones. http://www.ustr.gov/tpp 
sexta (Singapur), asomaron ciertos lineamientos de cómo se estructuraría un eventual tratado (25 capítulos) enfatizándose el problema de la coherencia regulatoria, aspecto que se repitió en la séptima ronda (Ciudad de Ho Chi Minh). En la octava (Chicago), reapareció la temática de la propiedad intelectual, lo que se explica por el número de grupos de interés (250) registrados como participantes. En la novena ronda (Lima), el gobierno estadounidense puso como tema de discusión el papel de las empresas estatales, asunto que particularmente es sensible para Vietnam. En la décima (Kuala Lumpur), no aparecieron temas novedosos.

La primera ronda de 2012 (Melbourne), reapareció públicamente, por la presencia de grupos de interés participantes, temas de propiedad intelectual (especialmente patentes farmacéuticas), inversiones y agricultura, de especial interés para Australia. Las siguientes tres rondas (Dallas, San Diego y Leesburg) se realizaron en EE.UU., con una gran visibilidad del tema de la propiedad intelectual y las normas laborales, asuntos muy presentes en los ACR de ese país. La última ronda de 2012 (Auckland) fue bastante significativa porque se trató de la primera en que oficialmente hubo 11 países negociadores, con la incorporación de México y Canadá. Además se dieron nuevos lineamientos de la forma que estaría adquiriendo el acuerdo, aumentando a 29 capítulos. Finalmente se informó que los temas que permanecerían abiertos serían telecomunicaciones, obstáculos técnicos al comercio y medidas fitosanitarias, además de otros asuntos no mencionados explícitamente (entre los que se deberían contar propiedad intelectual, agricultura y reglas de origen). En las dos rondas realizadas en 2013 no se presentaron diferencias significativas, mientras que en la última realizada a la fecha (Julio, en Kota Kinabalu, Malasia), se señalan avances en áreas que habían tenido un lugar secundario en anteriores rondas, como vestuario y agricultura, como asimismo se oficializa el ingreso de Japón como integrante número $12^{30}$.

Junto a lo informado en el marco de las rondas negociadoras, paralelamente ha ido apareciendo información por otras vías, como el caso de la Cumbre de Líderes de APEC 2011, realizada en Honolulú, Estados Unidos. En ella se detallaron los cinco grandes lineamientos en los cuales se estructuraría el TPP ${ }^{31}$ : Primero, un amplio y profundo acceso a mercados (incluyendo bienes, servicios e inversiones). Segundo, el carácter convergente del acuerdo, especialmente en la integración de la cadena de valor a través de la convergencia en las reglas de

30 "Statement on the 18th Round of Trans-Pacific Partnership Negotiations", Office of the United States Representative, www.ustr.gov/tpp

31 “Trans-Pacific Partnership (TPP). Trade Minister's Resport to Leaders”. Endorsed by TPP leaders, November 12, 2011, www.ustr.gov. 
origen. Tercero, la incorporación de nuevos temas, entre los que destaca la coherencia regulatoria (la relación con otros acuerdos pre-existentes y la implementación de nuevos estándares en los respectivos países). Cuarto, nuevos desafíos del comercio, que tiene que ver especialmente con la facilitación de inversiones y emprendimientos en nuevas tecnologías. Quinto, la flexibilidad del mismo, tanto en la incorporación de nuevas temáticas como en la entrada de nuevos integrantes.

Tomando en cuenta el tamaño de sus respectivas economías, resultan abrumadoras las diferencias entre EE.UU. y las restantes contrapartes, lo que hace inevitable pensar que un acuerdo necesariamente tendrá las características que representen en mayor medida los intereses estadounidenses. En diciembre de 2012, con 11 países en negociaciones (TPP11), EE.UU. representaba el 77\% del PIB conjunto, seguido en un lejano segundo lugar por Canadá 7\% y Australia, 5\%. Con el ingreso de Japón, la preponderancia de EE.UU. disminuye considerablemente a un $60 \%$ del PIB conjunto. En un escenario que considere el ingreso de Corea del Sur, que es una economía de gran relevancia, el PIB de la principal potencia del mundo representaría un 58\%. En cualquiera de los tres escenarios, de 2012 (TPP11), el actual (TPP12) y uno posible (TPP13), EE.UU. representa por sí solo más que la suma del PIB de los restantes países negociadores. (Cuadro 8).

Esta diferencia tan favorable a Estados Unidos hace necesario, por tanto, tener especialmente en cuenta los intereses de ese país en las negociaciones, puesto que probablemente la estructura de un posible acuerdo tendrá, probablemente, las características de un ACR en línea con los intereses estadounidenses. En ese sentido, EE.UU. tendría en las negociaciones una postura más aperturista en acceso a mercados en bienes y comercio de servicios y un especial interés en aquellos capítulos que buscan profundizar y consolidar internamente reformas económicas liberalizadoras en terceros países, como es política de competencia, inversión (protección), y el papel de las empresas estatales (Schott y Muir, 2012). Al mismo tiempo, tendría un especial énfasis en la protección de la Propiedad Intelectual, donde las negociaciones involucran básicamente dos grandes aspectos: la protección de derechos de autor y las patentes industriales. En el primer caso, busca imponer en sus negociaciones estándares más elevados que en la OMC (WTO Plus) o más allá que en ACR's anteriormente suscritos por EE.UU., lo que implicaría en algunos casos renegociar compromisos anteriores, como el caso del Acuerdo con Chile (Fergusson y Vaughn, 2011). En el caso de las patentes es muy probable un directo efecto en productos como los medicamentos, que tienen un alto impacto en políticas internas de alta sensibilidad, como la salud pública (Frankel, 2012). Junto con ello, buscaría incluir en los ACR's capítulos sobre Medio Ambiente y de Aspectos Laborales, que son temá- 
ticas presentes principalmente en acuerdos impulsados por ese país. A la inversa, es probable que EE.UU. muestre una postura más bien defensiva en la apertura a su sector agrícola, particularmente el azucarero y lácteo, como así también el acceso a productos pecuarios, específicamente carne bovina. Un segundo punto sería determinadas reglas de origen para manufacturas como textiles y productos electrónicos (Schott y Muir, 2012; Fergusson y Vaughn, 2011).

Cuadro 8: Participación en el PIB grupal de Países TPP (En porcentajes)

\begin{tabular}{llll}
\hline & TPP11 & TPP12 & TPP13 \\
\hline Australia & 4,8 & 3,8 & 3,6 \\
Brunei Darussalam & 0,1 & 0,0 & 0,0 \\
Canadá & 7,2 & 5,7 & 5,4 \\
Chile & 0,9 & 0,7 & 0,7 \\
Estados Unidos & 77,2 & 60,9 & 58,0 \\
Japón & -- & 21,2 & 20,2 \\
Malasia & 1,1 & 0,9 & 0,8 \\
México & 5,7 & 4,5 & 4,3 \\
Nueva Zelandia & 0,7 & 0,6 & 0,5 \\
Perú & 0,7 & 0,6 & 0,5 \\
Singapur & 1,0 & 0,8 & 0,8 \\
Vietnam & 0,5 & 0,4 & 0,4 \\
Corea del Sur & -- & -- & 4,6 \\
Total TPP11 & 100 & -- & -- \\
Total TPP12 & -- & 100 & 100 \\
Total TPP13 & -- & -- & \\
\hline
\end{tabular}

*Los porcentajes se calcularon en base al PIB en USD constantes de 2005.

Fuente: World Development Indicators, Banco Mundial. 
Junto con el análisis de los probables tópicos centrales de las negociaciones del TPP, es interesante observar los capítulos de una serie de ACR, porque permitirían dar una idea de cómo tomaría forma un eventual acuerdo. En primer lugar, se tomarán en cuenta los lineamientos que se plantearon en la Cumbre de Líderes de APEC 2011, a partir de la declaración oficial. Los otros ACR son el NAFTA, de ASEAN con Australia y Nueva Zelanda y el de EE.UU. con Corea del Sur (KORUS). Esta selección se explica porque el NAFTA se ha constituido como una suerte de modelo a seguir para otros acuerdos ${ }^{32}$; el de ASEAN con Australia y Nueva Zelanda, porque es el que más se parecería al Regional Comprehensive Economic Partnership (ASEAN+6), que es un proceso negociador entre naciones del ASEAN con Australia, Corea del Sur, China, Japón, India y Nueva Zelanda, y que es visto como el modelo alternativo al TPP, sin la presencia de EE.UU. ${ }^{33}$. Asimismo, es relevante considerarlo porque sigue los patrones de un esquema de integración más que de un área de libre comercio propiamente dicha (Lewis, 2011). En el caso del KORUS, al ser el último ACR de EE.UU., vendría a señalar los lineamientos posibles en el Trans-Pacific Partnership (Schott, et al., 2013). Al comparar los índices tentativos tanto de este ACR con otros acuerdos seleccionados (Cuadro 9), el más parecido sería el de EE.UU. con Corea del Sur, en línea con lo señalado por Schott, et al. (2013), mientras que las mayores diferencias se darían principalmente al comparar con las ampliaciones realizadas en la Asociación de Países del Sudeste Asiático (ASEAN).

32 Baccini, L., Dür, A., Elsig, M., \& Milwwicz, K. (2011).

33 "Asian Nations Plan Trade Bloc That, Unlike U.S.'s, Invites China", The New York Times, 20 de Noviembre de 2012,. www,nytimes.com 
Cuadro 9: Índices tentativos de TPP y otros ACR

\begin{tabular}{|c|c|c|c|c|c|}
\hline & & $\mathrm{TPP}^{8}$ & ASEAN* & NAFTA & KORUS \\
\hline 1 & Acceso a Mercados & $\mathrm{X}$ & $\mathrm{X}$ & $\mathrm{X}$ & $\mathrm{X}$ \\
\hline 2 & Reglas de Origen & $\mathrm{X}$ & $\mathrm{X}$ & $X$ & $\mathrm{X}$ \\
\hline 3 & Procedimientos Aduaneros & $\mathrm{X}$ & $\mathrm{X}$ & $\mathrm{X}$ & $\mathrm{X}$ \\
\hline 4 & Medidas Sanitarias y Fitosanitarias & $\mathrm{X}$ & $\mathrm{X}$ & $\mathrm{X}$ & $\mathrm{X}$ \\
\hline 5 & Obstáculos Técnicos al Comercio & $\mathrm{X}$ & $\mathrm{X}$ & $\mathrm{X}$ & $\mathrm{X}$ \\
\hline 6 & Contratación Pública & $\mathrm{X}$ & -- & $\mathrm{X}$ & $\mathrm{X}$ \\
\hline 7 & Propiedad Intelectual & $\mathrm{X}$ & $\mathrm{X}$ & $\mathrm{X}$ & $\mathrm{X}$ \\
\hline 8 & Defensa Comercial & $\mathrm{X}$ & $\mathrm{X}$ & $\mathrm{X}$ & $\mathrm{X}$ \\
\hline 9 & Política de Competencia & $\mathrm{X}$ & $\mathrm{X}$ & $\mathrm{X}$ & -- \\
\hline 10 & Comercio Transfronterizo de Servicios & $\mathrm{X}$ & $\mathrm{X}$ & $\mathrm{X}$ & $\mathrm{X}$ \\
\hline 11 & Servicios Financieros & $\mathrm{X}$ & $\mathrm{X}$ & $\mathrm{X}$ & $\mathrm{X}$ \\
\hline 12 & Entrada Temporal de Personas de Negocios & $\mathrm{X}$ & -- & $X$ & -- \\
\hline 13 & Telecomunicaciones & $\mathrm{X}$ & $\mathrm{X}$ & $X$ & $\mathrm{X}$ \\
\hline 14 & Comercio Electrónico & $\mathrm{X}$ & $\mathrm{X}$ & -- & $\mathrm{X}$ \\
\hline 15 & Inversiones & $\mathrm{X}$ & $\mathrm{X}$ & $\mathrm{X}$ & $\mathrm{X}$ \\
\hline 16 & Medio Ambiente & $\mathrm{X}$ & -- & -- & $\mathrm{X}$ \\
\hline 17 & Asuntos Laborales & $\mathrm{X}$ & -- & -- & $\mathrm{X}$ \\
\hline 18 & Cooperación & $\mathrm{X}$ & -- & -- & -- \\
\hline 19 & Coherencia Regulatoria & $\mathrm{X}$ & -- & -- & -- \\
\hline 20 & Pequeñas y medianas empresas & $\mathrm{X}$ & -- & -- & -- \\
\hline 21 & Competitividad & $\mathrm{X}$ & -- & -- & -- \\
\hline 22 & Desarrollo & $\mathrm{X}$ & -- & -- & -- \\
\hline 23 & Asuntos Institucionales & $X$ & -- & $X$ & $X$ \\
\hline 24 & Solución de Controversias & -- & -- & $\mathrm{X}$ & $\mathrm{X}$ \\
\hline 25 & Creación de Capacidad Comercial & $\mathrm{X}$ & -- & -- & -- \\
\hline 24 & Textiles & $\mathrm{X}$ & -- & $\mathrm{X}$ & $\mathrm{X}$ \\
\hline 25 & Agricultura & -- & -- & $\mathrm{X}$ & $\mathrm{X}$ \\
\hline 26 & Fármacos & -- & -- & -- & $\mathrm{X}$ \\
\hline 27 & Petroquímica básica & -- & -- & $X$ & -- \\
\hline
\end{tabular}

ASEAN* (ASEAN + Australia y Nueva Zelanda)

Fuente: Regional Trade Agreements Information System (RTA-IS), OMC. 


\section{ELEMENTOS POLITICOS EN EL TPP}

$\mathrm{Al}$ analizar un proceso negociador es necesario no sólo considerar las dimensiones estrictamente comerciales. Si bien en los puntos desarrollados anteriormente están presentes elementos de política pública (policy), sobre todo en materia regulatoria, es necesario considerarlos de una manera más explícita en su dimensión de politics. Junto a los países presentes en las negociaciones, destacan también los ausentes, siendo el más claro ejemplo en ese sentido el caso de China. En materia comercial, este país representa un porcentaje especialmente relevante para prácticamente todos los países, más allá incluso de un ámbito estrictamente regional $^{34}$ (Cuadro 10). Esta ausencia no se explica en absoluto por la falta de interés de gran parte de los países en negociar un ACR con China, tanto porque ya los tienen o se encuentran negociándolos, como es el caso de ASEAN+6. Por el contrario, el TPP tendría que ver con una forma de consolidar la presencia estadounidense en la región, siendo el aspecto comercial complementario a objetivos de tipo político y de seguridad (Feinberg, 2003). Como argumentan Capling y Ravenhill (2012), el interés principal de EE.UU. tiene que ver con la subordinación de los intereses comerciales a una agenda de seguridad (un elemento que es posible de encontrar en sus ACR, especialmente con países del Medio Oriente a los intereses estadounidenses de largo plazo en la región del Asia-Pacífico.

Cuadro 10: Importancia de China para países del TPP, 2012 (\%)

\begin{tabular}{lllllllllllllll}
\hline & AUS & BR & CAN & CL & US & JP & ML & MX & NZ & PER & SG & VT \\
\hline TC $^{9}$ & 24,1 & 4,6 & 7,9 & 20,7 & 14,9 & 19,7 & 13,8 & 8,4 & 15,9 & 15,9 & 10,5 & 18 \\
& & & & & & & & & & & & & \\
E & 29,6 & 2,7 & 4,5 & 23,3 & 7,7 & 18,1 & 12,6 & 1,5 & 15,4 & 15,3 & 10,8 & 12,0 \\
& & & & & & & & & & & & & \\
I & 18,4 & 11,4 & 11,1 & 18,2 & 19,0 & 21,3 & 15,1 & 15,4 & 16,4 & 16,7 & 10,3 & 23,3 \\
& & & & & & & & & & & & \\
\hline
\end{tabular}

*Los porcentajes de Perú y Vietnam corresponden a 2011.

Fuente: World Integrated Trade Solution (WITS), Banco Mundial.

34 No obstante, al ver los flujos comerciales hay que ser cuidadosos en el análisis. Por ejemplo, en el caso de Chile las exportaciones están fuertemente influidas por los precios extraordinariamente altos del cobre, que representa casi la mitad del valor de sus exportaciones y materia prima, siendo China el principal comprador. Para más detalle al respecto ver las estadísticas de la Comisión Chilena del Cobre, http://www.cochilco.cl 
Sin embargo, pese a que el TPP recoge básicamente el interés de EE.UU., resulta simplista considerar a este proceso de negociación como una suerte de imposición estadounidense de un determinado enfoque en torno al libre comercio. Entre los restantes países también hay elementos más allá de un mero alineamiento en torno a un enfoque norteamericano, lo que implica que en cada uno de ellos hay convicciones internas más profundas que hacen deseable, desde sus propias perspectivas, una eventual adhesión al TPP. Ello implica la necesidad de considerar en el análisis otro tipo de factores más allá de los meramente comerciales.

Una primera aproximación es posible encontrarla en la información que entregan indicadores como el Worldwide Governance Indicators (WGI), del Banco Mundial, que se basa en el estudio de seis dimensiones que indicarían grados de gobernanza, a partir de la recopilación y sistematización de bases de datos proporcionadas por 30 instituciones ${ }^{35}$. De estas, se seleccionarán tres categorías ${ }^{36}$. Dos de ellas se enfocan en torno a la efectividad del gobierno (Government Effectiveness y Regulatory Quality), por cuanto dan cuenta del interés de los gobiernos en tener o desarrollar una determinada institucionalidad, en la cual los ACR juegan un papel bastante importante, especialmente a través de compromisos del tipo WTO + y WTO-X. Asimismo, se tomará en cuenta la categoría "Estado de Derecho" (Rule of Law), porque a través de los ACR, de acuerdo a las categorías señaladas por Ravenhill, los países precisamente buscan consolidar internamente, a través de su incorporación en los ordenamientos jurídicos internos por medio de acuerdos internacionales, determinados procesos de reformas económicas que implican transformaciones institucionales como las dos anteriores.

35 La definición de gobernanza es tomada como aquellas instituciones y reglas bajo las cuales la autoridad es ejercida dentro de un país. Este concepto abarca seis temáticas, que a su vez se agrupan en tres categorías. Estas son las siguientes: a) "the process by which governments are selected, monitored and replaced", que reúne a "Voice and Accountability" (VA), "Political Stability and Absence of Violence/ Terrorism" (PV). b) "The capacity of the government to effectively formulate and implement sound policies", que reúne a "Government Effectiveness" (GE) y "Regulatory Quality" (RQ). c) "The respect of citizens and the state for the institutions that govern economic and social interactions among them", que a su vez incluye a "Rule of Law" (RL) y "Control of Corruption" (CC). La descripción de ésta metodología como asimismo el listado completo de las instituciones que proveen estas bases de datos puede encontrarse en Kaufmann, D., Kraay, A., \& Mastruzzi, M. (2010).

36 Se trata de Estado de Derecho (Rule of Law), Efectividad del Gobierno (Government Effectiveness) y Calidad Regulatoria (Regulatory Coherence). Las definiciones exactas de cada una de éstas se encuentran en The Worldwide Governance Indicators, update 2012, base de datos disponible en www.govindicators.org. 
En relación a la "Efectividad del Gobierno", que refleja la calidad del sector público y la credibilidad de éste en la implementación de políticas, en general los indicadores son bastante elevados, ubicándose en su mayoría en el quintil superior (sobre el 80\%), salvo Brunei Darussalam, que se ubica muy cercano a ese umbral, y México, que se ubica en el percentil 64, sobre la media. Las excepciones más relevantes serían Perú y Vietnam, con bajos índices en éste ítem (Cuadro 11). En el caso de la "Calidad Regulatoria", que muestra la capacidad del gobierno en su conjunto para formular e implementar políticas que promuevan el desarrollo del sector privado, cada uno de los países se ubica en percentiles similares a los de la categoría anterior, salvo en el caso de Perú, que aumenta en 20 puntos y Vietnam, que desciende al percentil 29.

Finalmente en "Estado de Derecho", que refleja la percepción respecto al respeto de la propiedad privada y de los contraltos que regulan su funcionamiento, el patrón se repite, salvo en el caso de Perú que vuelve a descender a un bajo nivel, en el percentil 29, siendo más bajo incluso que Vietnam, que se ubica en lugar $40 \mathrm{o}$ incluso de México, en el 38, que puede entenderse por la delicada situación de ese país por la violencia generada en el marco de la lucha contra el narcotráfico (Cuadro 11). En su conjunto, estos resultados muestran en general determinadas formas comunes en cómo se organiza el Estado, las que cuentan con estándares relativamente similares, los que buscan de alguna manera ser confirmados en el marco del TPP, sobre todo por aquellos capítulos que apuntan a aspectos regulatorios, como los de protección a la propiedad intelectual o de inversiones, contratación pública y política de competencia. En el caso específico de Perú y Vietnam, además con ciertos matices el de México, un eventual acuerdo vendría a representar una forma de obligar a estos países a implementar políticos que fueran en la dirección de acercarse a los estándares del resto de los países. 
Cuadro 11: Calidad Institucional de países TPP, según ubicación en percentiles (0 más bajo y 100 más alto), 2012

\begin{tabular}{llll}
\hline & Efectividad del Gobierno & Calidad Regulatoria & Estado de Derecho \\
\hline Australia & 95 & 97 & 96 \\
Brunei & 77 & 85 & 78 \\
Darussalam & & 96 & 95 \\
Canadá & 97 & 93 & 88 \\
Chile & 84 & 92 & 91 \\
EE.UU. & 89 & 78 & 87 \\
Japón & 88 & 74 & 66 \\
Malasia & 81 & 61 & 38 \\
México & 64 & 100 & 99 \\
Nueva & 98 & 69 & 33 \\
Zelanda & 49 & 97 & 93 \\
Perú & 99 & 29 & 40 \\
Singapur & 45 & & \\
Vietnam & 49 & 93 \\
\hline
\end{tabular}

Fuente: "The Worldwide Governance Indicators, update 2012".

Junto con la coincidencia en cuanto a la forma en cómo se concibe la organización del Estado, dentro del esquema de economías que comparten una cierta visión de modelos de desarrollo económico basados en el libre mercado, es necesario considerar aspectos de tipo ideológico, en el sentido ya no de prácticas en materia de políticas públicas, sino con el soporte doctrinario a este tipo de concepciones. Ello debido a que el TPP representa un proyecto entre países afines, fundamentalmente de sus respectivas élites, que son las que buscan e impulsan este tipo de acuerdos. Al respecto, resulta interesante analizar los resultados del Índice de Libertad Económica (Index of Economic Freedom, IEF ${ }^{37}$ ), realizado desde 1995 por dos instituciones identificadas con el pensamiento neoliberal ${ }^{38}$, como The Heritage Foundation y el periódico The Wall Street Journal. Este indicador es uno de los que se consideran para la realización del Worldwide Governance Indicators, y la razón de seleccionarlo reside en que mide aspectos de tipo doctrinario. Éste consiste en la medición de diez indicadores que revelarían el grado de libertad

\footnotetext{
37 Index of Economic Freedom. http://www.heritage.org/index/

38 Crouch, C. (2011).
} 
económica de una serie de países. Éstos se agrupan en cuatro categorías ${ }^{39}$. Para efectos del presente artículo, se analizarán los resultados de la categoría apertura de mercados, que agrupa al grado de liberalización en materia comercial, de inversiones y del sector financiero. Los resultados obtenidos, en una escala de 1 a 100, se clasifican de la siguiente manera: "libre" (100-80); "mayoritariamente libre" (79,9-70); "moderadamente libre" (69.9-60); "mayoritariamente no libre" (59.9-50); "represivo" (49.9-0).

Los resultados del último estudio (2013), muestran que el TPP es, a grandes líneas, el club de los que hacen bien las tareas, parafraseando la crítica que hizo Stiglitz respecto a países que cumplieron, al pie de la letra, las indicaciones de organismos como el Fondo Monetario Internacional, erróneamente desde su perspectiva (Stiglitz, 2012).

Siete de 11 de los países negociadores ${ }^{40}$ son considerados "libres" o "mayoritariamente libres", ubicándose más de la mitad de ellos (seis) en los primeros 10 puestos del listado. Si bien tres de ellos se ubican como "moderadamente libres" (México, Malasia y Perú), su promedio los ubica muy cerca del umbral para ser considerado "mayoritariamente libre" (70 puntos). Sólo Vietnam es clasificado como "mayoritariamente no libre", aunque sin embargo ha avanzado más de diez puntos desde $1995^{41}$ (Cuadro 12).

$\mathrm{Al}$ analizar los resultados de la categoría "Apertura de Mercados", los resultados en el caso del comercio son aún más decidores respecto a que el TPP es un club de países con una visión bastante en común respecto del libre comercio: Nueve de 11 países son considerados como libres y dos de ellos, incluyendo a Vietnam, como "mayoritariamente libres" (Cuadro 12). Los resultados en las otras dos categorías (inversiones y sector financiero), son un reflejo de aquellas áreas en donde se ve una mayor probabilidad de desacuerdos, como son la protección a la inversión extranjera y la regulación al sector financiero.

39 Rule of Law (property rights; freedom from corruption); Limited Government (fiscal freedom, government spending); Regulatory Efficency (bussines freedom, labor freedom, monetary freedom); Open Markets (trade freedom, investment freedom, financial freedom).

41 En 1995 Vietnam obtuvo 41.7 puntos, ubicándolo como un país "represivo" de la libertad económica. Fuente: Index of Economic Freedom, http://www.heritage.org/index/ 
Cuadro 12: Índice de Libertad Económica 2013, según apertura de mercados.

\begin{tabular}{llllll}
\hline & $\begin{array}{l}\text { Resultados } \\
\text { Generales }\end{array}$ & $\begin{array}{l}\text { Apertura de } \\
\text { Mercados }\end{array}$ & & & \\
\hline & $\begin{array}{l}\text { Posición en el } \\
\text { Ranking }\end{array}$ & $\begin{array}{l}\text { Promedio } \\
\text { General }\end{array}$ & Comercio & Inversiones & $\begin{array}{l}\text { Sector } \\
\text { Financiero }\end{array}$ \\
\hline AUS & 2 & 82.6 & 86.2 & 80 & 90 \\
CAN & 6 & 79.4 & 88.2 & 75 & 80 \\
CL & 7 & 79 & 82 & 85 & 70 \\
US & 10 & 76.7 & 86.4 & 70 & 70 \\
JP & 24 & 71.8 & 81.8 & 60 & 50 \\
ML & 56 & 66.1 & 77 & 45 & 50 \\
MX & 50 & 67.0 & 80.6 & 70 & 60 \\
NZ & 4 & 81.4 & 86.8 & 80 & 80 \\
PER & 44 & 68.2 & 85 & 70 & 60 \\
SG & 3 & 88 & 90 & 75 & 80 \\
VT & 140 & 51 & 78.6 & 30 & 30 \\
\hline
\end{tabular}

Fuente: Index of Economic Freedom.

\section{CONCLUSIONES}

El TPP es ante todo un proceso de negociaciones comerciales entre 12 países de Asia - Pacífico y América que comparten una determinada visión en cuanto a sus modelos de desarrollo económico, donde la creencia de una apertura comercial hacia sus respectivos mercados externos es un aspecto central. Sin embargo, como se ha señalado anteriormente, un ACR no necesariamente involucra aspectos relacionados exclusivamente con el comercio, sino que tras de él están presentes visiones respecto a aspectos más bien políticos, como son las definiciones respecto a una visión de cómo ordenar sus principales directrices en materia de política económica ${ }^{42}$, los que a su vez son elementos de gran importancia en sus

42 En ese sentido, es útil el enfoque propuesto desde el Nuevo Institucionalismo, en que el ordenamiento en torno a una manera de organizar la economía es producto de la confrontación/interacción de intereses sociales y políticos sobre maneras de interpretarla, donde el papel de la acción política, tanto en las ideas como en las instituciones, juegan un papel central. Ver Hall, P. (1987). The movement from Keynesianism to monetarism: Institutional analysis and British economic policy in the 1970s. 
respectivas definiciones e intereses en materia de política, incluyendo por cierto la exterior, donde la regulación de la apertura comercial juega un papel central.

Sin duda, en una negociación de un ACR la liberalización regulada del comercio es un elemento de gran importancia. Como muestran los datos, existen importantes potenciales incrementos en el comercio intrabloque entre los miembros de este eventual pacto, siendo el espacio donde eventualmente podrían estar en un mismo acuerdo países como Japón o EE.UU., aunque al mismo tiempo, la presencia de ambos podría hacer aún más difíciles las negociaciones, que ya presentan complicaciones en temas como el comercio agrícola y los estándares de Propiedad Intelectual. De prosperar estas negociaciones, que ya llevan un importante recorrido, estaríamos en presencia del mayor bloque comercial posible, en un escenario en que el Transantlantic Trade and Investment Partnership (TTIP), entre la Unión Europea y Estados Unidos son conversaciones de una complejidad tanto o mayor que el TPP.

Estos elementos sin duda se encuentran presentes en este caso, que se da en un área del mundo de creciente importancia, en cuanto a su dinamismo económico pero que a la vez ha sido considerada de relevancia en términos políticos. Si bien se trata de una negociación entre 12 países, las características del TPP, tanto por la diferencia abismante entre EE.UU. y el resto de los países presentes en relación al peso de sus economías, como asimismo de las temáticas presentes en las rondas negociadoras, hacen presente la situación que en realidad éste sea un Acuerdo de EE.UU. para el Asia-Pacífico. No obstante, es incorrecto señalar, pese a los evidentes intereses estadounidenses, que se trataría de una suerte de reedición de una política aplicada por ese país al estilo de las implementadas especialmente en América Central en la primera mitad del siglo XX. En ese sentido, más que una diplomacia del cañonero, lo que se observa es una coincidencia de países que comparten este proyecto concebido en términos estadounidense o al menos, parecen dispuestos a pagar un precio elevado, en cuanto al nivel de compromisos contenidos en el TPP, por el acceso preferencial al mercado de EE.UU.

Al respecto, los indicadores macroeconómicos respecto al modo en que miembros del TPP entienden el ordenamiento económico tienden a coincidir, en el sentido de que es un grupo de países donde tiene peso un modelo de libre mercado, como lo muestra el ranking del IEF. Asimismo, si bien se presentan diferencias importantes en el cómo se expresa institucionalmente esta visión, que se refleja en los datos del WGI, los acuerdos comerciales no sólo buscan negociar la apertura en bienes y servicios, sino que tras ellos se contienen una serie de regulaciones que necesariamente implicarán cambios regulatorios y de diseño institucional en los países que actualmente presentan indicadores más bajos en esta materia. Si 
tomamos en cuenta las características que presentaría el TPP, que se asemejan al KORUS, el argumento respecto al impacto en la institucionalidad política de esta negociación se refuerza (Aaronson, Abouharb, y Rodwan, 2013).

Un punto que es necesario tomar en cuenta, a partir de lo expuesto en el presente artículo, es el contexto en que se producen estas negociaciones, con un desgaste prolongado, de casi nueve años, en las negociaciones a nivel multilateral, que comenzaron en 2001. Ello coexiste con un fenómeno profundo de negociaciones paralelas a la OMC, como es el fenómeno de los ACR. El mismo proporciona elementos que posibilitan un amplio margen para que los países, con el TPP, entren en una nueva fase, como son los denominados mega-bloques, donde para el caso de los países latinoamericano que tienen más internalizado un esquema de libre mercado, el costo de quedar fuera es un elemento que aparece presente, y eventualmente, acentúe las diferencias con aquellas naciones de la región que se mantengan al margen (Rosales y Herreros, 2014).

Pese a la importancia del TPP, los países continúan utilizando en paralelo las negociaciones bilaterales, incluso entre países que participan de estas negociaciones. Si bien una interpretación posible es que esta situación pueda explicarse por una expectativa de fracaso del TPP, y por tanto buscar vías alternativas, en realidad lo que parece mostrar la información acá presentada corresponde más bien a un pragmatismo de los respectivos países.

Las características de este proceso negociador, como la flexibilidad en la incorporación de nuevas partes y la profundidad en el grado de compromisos que se pretenden adquirir en caso de un final exitoso del TPP, hacen precisamente poner un signo de interrogación respecto al desenlace de las negociaciones. Las negociaciones en materias como la protección a la propiedad intelectual y a las inversiones, que pueden llevar incluso a renegociar anteriores ACR de EE.UU., además de la complicación de las conversaciones en materias agrícolas (la presencia de Japón, muy proteccionista en ese sector), sin duda no son elementos facilitadores. Pese a lo anterior, no habría opción de una versión light del TPP, en el sentido de sacrificar nivel compromisos en función de lograr un acuerdo entre 12 miembros. Efectivamente, este proceso negociador es en realidad el proyecto de EE.UU. para 12 países de la cuenca del Pacífico, donde si bien la dinámica es más bien con elementos de un Acuerdo del Siglo XXI, en realidad siguen estando presentes elementos importantes de un modelo de la centuria anterior. 


\section{REFERENCIAS BIBLIOGRAFICAS}

\section{$\underline{\text { Fuentes Primarias }}$}

Index of Economic Freedom. http://www.heritage.org/index/

Regional Trade Agreements Information System (RTA-IS), World Trade Organization (Australia). Department of Foreign Affairs and Trade. Recuperado de http://www.dfat.gov.au

Ministry of Foreign Affairs and Trade. Brunei. Recuperado de http://www.mofat. gov.bn

Dirección de Relaciones Económicas Internacionales, Ministerio de Relaciones Exteriores. Chile.

Ministry of Foreign Affairs and International Trade. Canadá. Recuperado de www.international.gc.ca

Office of the United States Trade Representative. EE.UU. Recuperado de www. ustr.gov

Ministry of Foreign Affairs. Japan. Recuperado de http://www.mofa.go.jp/

Ministry of International Trade and Industry. Malasia. Recuperado de www.miti. gov.my

Secretaría de Economía. México. Recuperado de www.economia.gob.mx

Ministry of Foreign Affairs and Trade. Nueva Zelanda. Recuperado de http:// www.mfat.govt.nz

Acuerdos Comerciales del Perú. Perú. Recuperado de www.acuerdoscomerciales.gob.pe Singapore FTA. Singapur. Recuperado de www.fta.gov.sg

Ministry of Industry and Trade. Vietnam. Recuperado de www.moit.gov.vn/

\section{$\underline{\text { Fuentes Secundarias }}$}

Aaronson, S. A. \& Abouharb, M. R. (2013). Does the WTO help member states improve governance? World Trade Review, 1-36.

Baccini, L., Dür, A., Elsig, M., \& Milwwicz, K. (2011). The Design of Preferential Trade Agreements: A New Dataset in The Making. Staff Working Paper ERSD -2011-10. Economic Research and Statistic Division, World Trade Organization.

Baldwin, R. (1993). A Domino Theory of Regionalism. National Bureau of Economic Research. Working Paper No 4465

Bhagwatti, J. (2008). Termites in the Trading System. How preferential Trade Agreements Undermine Free Trade. Oxford: Oxford University Press. 
Capling, A. \& Ravenhill J. (2012). Multilateralizing Regionalism or Securitization?. En Lim. C. L, Elms, D. K., \& Low, P. (Eds.). The Trans-Pacific Partnership. A quest for a Twenty-first Century Trade Agreement. Cambridge: Cambridge University Press.

Carpenter, T. (2008). A historical perspective on Regionalism. En Baldwin, R., \& Low, P. (Eds.) Multilateralizing Regionalism. Cambridge: Cambridge University Press.

Crouch, C. (2001). The Strange Non-Death of Neoliberalism. United Kingdom: Polity Press.

Elms, D. K., \& Low, P. (Eds.) (2013). Global value chains in a changing world. World Trade Organization/ Fung Global Institute/ Tomasek Foundation for Trade \& Negotiations.

Elsig, M. (2007). The EU's Choice of Regulatory Venues for Trade Negotiations: A Tale of Agency Power? Journal of Common Market Studies, (4).

Feinberg, R. (2003). The Political Economy of United States Free Trade Arrangements. World Economy, (26), 77.

Fergusson, I., \& Vaughn, B. (2011). The Trans-Pacific Partnership Agreement. Congressional Research Service, R4052. Recuperado de www.crs.gov

Frankel, S. (2012). The Intellectual Property Chapter in the TPP. En Lim. C. L, Elms, Deborah K., \& Low, P. (Eds.). The Trans-Pacific Partnership. A quest for a Twenty-first Century Trade Agreement. Cambridge: Cambridge University Press.

Gallagher, P. (2005). The First Ten Years of the WTO 1995-2005. Cambridge: Cambridge University Press.

Goode, W. (2005). Dictionary of Policy Trade Terms. Cambridge University Press. Hall, P. (1987). The movement from Keynesianism to monetarism: Institutional analysis and British economic policy in the 1970s. En Steinmo S., Thelen K., \& Longstreth F. Structuring politics Historical Institutionalism in Comparative Analysis. Cambridge: Cambridge University Press.

Horn, H., Mavroidis, P., \& Sapir, A. (2009) Beyond the WTO? An Anatomy of EU and US preferential trade agreements. Bruegel Blueprint Series.

Kaufmann, D., Kraay, A., \& Mastruzzi, M. (2010). The Worldwide Governance Indicators. Methodology and Analytical Issues. Policy Research Working Paper 5430. The World Bank.

Debería Lewis, M. K. (2011). The Trans-Pacific Partnership: New Paradigm or Wolf in Sheep's Clothing? Boston College International \& Comparative Law Review, 34(1), 27-52.

Liang, M. (2012). Trade negotiations: rules of origin. En Lim. C. L, Elms, D. K., \& Low, P. (Eds.) The Trans-Pacific Partnership. A quest for a Twenty-first Century Trade Agreement. Cambridge University Press.

Lim. C. L, Elms, D. K., \& Low, P. (Eds.) (2012). The Trans-Pacific Partnership. A quest for a Twenty-first Century Trade Agreement. Cambridge University Press. 
Low, P. (2012). The TPP in a Multilateral World. En Lim. C. L, Elms, D. K., \& Low, P. (Eds.). The Trans-Pacific Partnership. A quest for a Twenty-first Century Trade Agreement. Cambridge University Press.

Organización Mundial del Comercio (OMC). (2003). Los textos jurídicos. Los resultados de la Ronda Uruguay de Negociaciones Comerciales Multilaterales. Organización Mundial del Comercio. Ginebra: Autor

Organización Mundial del Comercio (OMC). (2011).La OMC y los acuerdos comerciales preferenciales: de la coexistencia a la coherencia. Informe de Comercio Mundial. Organización Mundial de Comercio. Ginebra: Autor

Ravenhill, J. (2011). Regional Trade Agreements. En Ravenhill, J. (Ed.). Global Political Economy. Oxford University Press. Pp.179-185.

Rosales, O., \& Herreros, S. (2014). Mega-regional trade negotiations: what is at stake for Latin America? Working Paper. Inter-American Dialogue, January.

Schott, J., Kotschwar, B., \& Muir, J. (2013). Understanding the Trans-Pacific Partnership. Policy Analysis in International Economics. Peterson Institute for International Economic.

Schott, J. \& Muir, J. (2012). US PTA's: what's been done and what it means for the TPP negotiations. En Lim. C. L, Elms, D. K., \& Low, P. (Eds.). The TransPacific Partnership. A quest for a Twenty-first Century Trade Agreement. Cambridge: Cambridge University Press.

Stiglitz, J. (2002). La Globalización y sus descontentos. Buenos Aires: Taurus. Wilhelmy, M. (2007). Beyond P4. New Zealand International Review. (32), 2. Williamson, J. (2004). A short history of the Washington Consensus. Conferencia en Fundación CIDOB, Barcelona, 24-25 de Septiembre de 2004. Recuperado de www.piie.com 